\title{
CLOSED COVERINGS IN ČECH HOMOLOGY THEORY
}

BY

E. E. FLOYD

1. Introduction. We treat here such topics as homology local connectedness, regular convergence, and the Vietoris mapping theorem $\left.{ }^{1}\right)$. In general, we are interested in those topics of topology which are based on the technique of chain-realizations. We present an alternative technique, based on one central theorem (Theorem 2.3). One justification for our technique is that it allows the use of compact coefficient groups as well as fields. Moreover, we hope that with further study it will provide a certain amount of unification of the topics treated.

The paper is divided into two parts. The first part consists of $\S \S 2,3$, and 4 . In $\S 2$ we state the basic theorem and some of its corollaries. In $\S \S 3$ and 4 we give a few applications of our theorem to the topics already noted. In the second part, which is not dependent on the first part, we prove the basic theorem. In $\$ \S 5$ and 6 we develop in detail a Kelley-Pitcher theory of finite closed coverings of compact spaces. This theory was partially developed in the well-known Kelley-Pitcher paper on exact sequences [3, pp. 703-706]. In $\$ 7$ we use this development to prove the basic theorem. We would be interested in knowing whether or not the basic theorem can be proved by more elementary means.

2. The basic theorems. In this paper, a space will always be a Hausdorff space. Suppose that $\alpha=\left(A^{1}, \cdots, A^{r}\right)$ is an ordered, finite covering of a space $X$. In $r$-space, let $\tilde{A}^{i}$ be the point whose $j$ th coordinate is $\delta_{j}^{i}$. Then the nerve of $\alpha$ will be the collection of all simplices $\left(\widetilde{A}^{i_{0}}, \ldots, \widetilde{A}^{i_{q}}\right)$ with $A^{i_{0}} \cap \ldots$ $\cap A^{i_{q}} \neq \varnothing$. If $X$ is compact, $H_{n}(X)$ will denote the $n$-dimensional Cech homology group with coefficients in a fixed group $\$$, which may be either a field or a compact abelian group; $H_{0}(X)$ will denote the reduced 0 -dimensional group. For a covering $\alpha, H_{n}(\alpha)$ denotes the homology group of the nerve of $\alpha$.

If $u=\left(U^{1}, \cdots, U^{r}\right)$ is an open covering of $X$, there is the projection homomorphism $\pi_{u}: H_{n}(X) \rightarrow H_{n}(u)$ which assigns to each element of $H_{n}(X)$ its $u$-coordinate.

By a closed covering $\alpha$ of a compact space $X$, we will always mean an ordered, finite covering $\alpha=\left(A^{1}, \cdots, A^{r}\right)$ by closed sets in $X$ such that every point of $X$ is in the interior of some $A^{i}$. Given such an $\alpha$, there exists an open covering $u=\left(U^{1}, \cdots, U^{r}\right)$ of $X$ such that $U^{i} \supset A^{i}$ and $U^{i_{0}} \cap \cdots \cap U^{i_{q}} \neq \varnothing$

Presented to the Society, April 20, 1956; received by the editors December 11, 1955.

(1) For bibliographical notes concerning homology local connectedness, see Wilder [6]; for a bibliography on regular convergence, see White [4]; for a modern treatment of the Vietoris mapping theorem, see Begle [1]. 
if and only if $A^{i_{0}} \cap \cdots \cap A^{i_{q}} \neq \varnothing$ (this is a special case of (6.1)). Then $u$ and $\alpha$ have the same nerve and $H_{n}(\alpha)=H_{n}(u)$. Define a projection homomorphism $\pi_{\alpha}: H_{n}(X) \rightarrow H_{n}(\alpha)$ by $\pi_{\alpha}=\pi_{u}$. It may be checked that $\pi_{\alpha}$ is independent of $u$.

If $\alpha$ and $\beta$ are finite collections of closed subsets of $X$, then $\beta$ refines $\alpha$, or $\beta>\alpha$, if and only if given an element $B$ of $\beta$ there exists an element $A$ of $\alpha$ with $B C A$. A projection $\pi_{\beta \alpha}$ assigns to each $B$ in $\beta$ such an $A$. The induced homomorphism of $H_{n}(\beta)$ into $H_{n}(\alpha)$ will also be denoted by $\pi_{\beta \alpha}$ (or occasionally by just $\pi$ ). If $\alpha$ and $\beta$ are closed coverings of $X$ with $\beta>\alpha$, it may be checked that $\pi_{\beta \alpha} \pi_{\beta}=\pi_{\alpha}$.

If $A$ and $B$ are closed subsets of $X$ with $A \subset B$, we denote by $I_{A B}: H_{n}(A)$ $\rightarrow H_{n}(B)$, or occasionally by $I$, the injection homomorphism which is induced by inclusion. We denote by $H_{n}(A ; B)$ the image of $I_{A B}$.

(2.1) Definition. If $\alpha$ and $\beta$ are finite collections of closed subsets of $X$ and $n$ is a non-negative integer, we write $\beta^{n}>\alpha(\beta$-refines $\alpha)$ if and only if given an element $B$ of $\beta$ there exists an element $A$ of $\alpha$ with $B \subset A$ and $H_{j}(B ; A)=0$ for all $j \leqq n$.

We write $\beta^{n} \gg \alpha$ ( $\beta$ strongly $n$-refines $\alpha$ ) if and only if $\beta>\alpha$ and there exists a projection $\pi_{\beta \alpha}: \beta \rightarrow \alpha$ such that $H_{j}\left(B^{i_{0}} \cap \cdots \cap B^{i_{q}} ; \pi B^{i_{0}} \cap \cdots \cap \pi B^{i_{q}}\right)=0$ for all $B^{i_{0}}, \cdots, B^{i_{q}}$ in $\beta$ and all $j \leqq n$.

(2.2) If $\gamma^{n}>\beta$ and $\beta$ star-refines $\alpha$, then $\gamma^{n} \gg \alpha$.

Proof. Since $\gamma^{n}>\beta$, there exists a projection $\pi^{\prime}: \gamma \rightarrow \beta$ such that $H_{j}\left(C ; \pi^{\prime} C\right)$ $=0$ for each $C$ in $\gamma$ and each $j \leqq n$. Since $\beta$ star-refines $\alpha$, there is a projection $\pi^{\prime \prime}: \beta \rightarrow \alpha$ such that if $B$ is in $\beta$ then every element of $\beta$ which intersects $B$ is in $\pi^{\prime \prime} B$. Let $\pi=\pi^{\prime \prime} \pi^{\prime}$. Suppose $C^{i_{0}} \cap \cdots \cap C^{i_{q}} \neq \varnothing$. Then

$$
C^{i_{0}} \cap \cdots \cap C^{i_{q}} \subset C^{i_{0}} \subset \pi^{\prime} C^{i_{0}} \subset \pi C^{i_{0}} \cap \cdots \cap \pi C^{i_{Q}} .
$$

Then $H_{j}\left(C^{i_{0}} \cap \cdots \cap C^{i_{q}} ; \pi C^{i_{0}} \cap \cdots \cap \pi C^{i_{q}}\right)=0$ since $H_{j}\left(C^{i_{0}} ; \pi^{\prime} C^{i_{0}}\right)=0$, $j \leqq n$.

The following is the basic theorem of the paper; its proof is deferred to $\S 7$.

(2.3) THEOREM. Suppose that $A_{-1} \supset A_{0} \supset \cdots \supset A_{n}$ is a sequence of closed subsets of a compact space $X$, and that $\alpha_{-1} \ll^{n} \alpha_{0} \ll^{n} \cdots \ll^{n} \alpha_{n}$, where $\alpha_{i}$ is a closed covering of $A_{i}$. Then

(i) the kernel of $\pi_{\alpha_{n}}: H_{j}\left(A_{n}\right) \rightarrow H_{j}\left(\alpha_{n}\right)$ is contained in the kernel of the injection $I: H_{j}\left(A_{n}\right) \rightarrow H_{j}\left(A_{-1}\right)$ for all $j \leqq n$, and

(ii) the image of $\pi_{\alpha_{n}, \alpha_{0}}: H_{j}\left(\alpha_{n}\right) \rightarrow H_{j}\left(\alpha_{0}\right)$ is contained in the image of the projection $\pi_{\alpha_{0}}: H_{j}\left(A_{0}\right) \rightarrow H_{j}\left(\alpha_{0}\right)$ for all $j \leqq n+1$.

We now study the implications of the theorem in case $A_{i}=X$, all $i$.

(2.4) Definition. Let $\alpha, \beta$ be closed coverings of the compact space $X$. We say that $\alpha, \beta$ determine $H_{n}(X)$ if and only if $\alpha<\beta$ and $\pi_{\alpha}: H_{n}(X) \rightarrow H_{n}(\alpha)$ maps $H_{n}(X)$ isomorphically onto the image of the projection $\pi_{\beta \alpha}: H_{n}(\beta)$ $\rightarrow H_{n}(\alpha)$. We say that $\alpha, \beta$ partially determine $H_{n}(X)$ if and only if image 
$\pi_{\alpha}=$ image $\pi_{\beta \alpha}$ (this latter is equivalent to the classical notion that $\beta$ is a normal refinement $[7$, p. 140] of $\alpha$ ).

(2.5) If $\alpha, \beta$ (partially) determine $H_{n}(X)$ and $\gamma>\beta$, then $\alpha, \gamma$ (partially) determine $H_{n}(X)$.

Proof. It is always true that image $\pi_{\alpha} \subset$ image $\pi_{\gamma \alpha}$. But image $\pi_{\gamma \alpha} \subset$ image $\pi_{\beta \alpha}=$ image $\pi_{\alpha}$, so image $\pi_{\alpha}=$ image $\pi_{\gamma \alpha}$.

(2.6) If $\beta, \gamma$ partially determine $H_{n}(X)$ and if $\beta>\alpha$ then $\alpha, \gamma$ partially determine $H_{n}(X)$.

Proof. We have image $\pi_{\gamma \alpha}=\pi_{\beta \alpha}$ (image $\left.\pi_{\gamma \beta}\right)=\pi_{\beta \alpha}$ (image $\pi_{\beta}$ ) =image $\pi_{\alpha}$.

The following consequence of (2.3) is used as the basis for the rest of the paper.

(2.7) THEOREM. If $X$ is a compact space and if $\alpha_{-1} \ll^{n} \alpha_{0} \ll^{n} \ldots \ll^{n} \alpha_{2 n}$, where $\alpha_{i}$ is a closed covering of $X$, then $\alpha_{n}, \alpha_{2 n}$ determine $H_{j}(X)$, for all $j \leqq n$, and partially determine $H_{n+1}(X)$.

Proof. In (2.3 i), let $A_{i}=X$. Hence according to $(2.3 \mathrm{i})$ the kernel of $\pi_{\alpha_{n}}$ is contained in the kernel of the identity map of $H_{j}(X), j \leqq n$, and hence $\pi_{\alpha_{n}}$ is an isomorphism into for $j \leqq n$. In (2.3 ii), set $A_{i}=X$ and consider the coverings $\alpha_{n}, \cdots, \alpha_{2 n}$. In (2.3 ii), the image of $\pi_{\alpha_{2 n}, \alpha_{n}}: H_{j}\left(\alpha_{2 n}\right) \rightarrow H_{j}\left(\alpha_{n}\right)$ is contained in the image of $\pi_{\alpha_{n}}$ for $j \leqq n+1$. Since the opposite inclusion always holds, the two images are equal. Hence $\alpha_{n}, \alpha_{2 n}$ partially determine $H_{j}(X)$ for $j \leqq n+1$. The theorem follows.

3. Locally connected space; the Vietoris mapping theorem. We consider here a few properties of locally connected spaces; these are known for the case when the coefficient group is a field [7, Chap. 6]. On the basis of these, we give a new proof of the Vietoris mapping theorem as given by Begle [1].

(3.1) Definitron. A compact space $X$ is said to be $l c^{n}, n$ a non-negative integer, if and only if given $x \in X$ and a closed neighborhood $U$ of $x$, there exists a closed neighborhood $V$ of $x$ with $H_{j}(V ; U)=0$, all $j \leqq n$.

(3.2) If $X$ is an $c^{n}$, compact space, then given a closed covering $\alpha$ of $X$, there exists a closed covering $\beta$ of $X$ with $\beta^{n} \gg \alpha$.

Proof. Suppose that $\gamma$ is a closed covering which star-refines $\alpha$. For each $x \in X$, there is an element $C$ of $\gamma$ which contains $x$ in its interior. Hence there is a closed neighborhood $V_{x}$ of $x$ with $H_{j}\left(V_{x} ; C\right)=0$ for $j \leqq n$. Let $\beta$ be a finite collection of the $V_{x}$ whose interiors cover $X$. Then $\beta^{n}>\gamma$ and $\gamma$ star-refines $\alpha$. By (2.2), $\beta^{n} \gg \alpha$.

The following is equivalent to a classical theorem $[7$, p. 180] when the coefficient group is a field.

(3.3) Theorem. Suppose that $X$ is a compact $l c^{n}$ space. For each sufficiently small covering $\alpha$ of $X$, there exists a closed covering $\beta>\alpha$ such that $\alpha, \beta$ determine $H_{j}(X)$, all $j \leqq n$, and partially determine $H_{n+1}(X)$.

Proof. Let $\alpha_{-1}$ be a closed covering of $X$. By (3.2), there are closed cover- 
ings $\alpha_{0}, \cdots, \alpha_{n-1}$ with $\alpha_{-1} \ll^{n} \ldots \ll^{n} \alpha_{n-1}$. By (3.2), for all sufficiently small $\alpha_{n}$ we have $\alpha_{n}^{n} \gg \alpha_{n-1}$. Given such an $\alpha_{n}$, we get a sequence $\alpha_{-1} \ll^{n} \cdots \ll^{n} \alpha_{2 n}$. By (2.7), $\alpha_{n}, \alpha_{2 n}$ determine $H_{j}(X), j \leqq n$, and partially determine $H_{n+1}(X)$.

(3.4) Definition. We shall say that the coefficient group $G$ is elementary if it is either a field or an elementary compact group $\left({ }^{2}\right)$. In case $G$ is elementary, we say that $H_{n}(X)$, or a subgroup thereof, is elementary if it is a finite dimensional vector space when $G$ is a field, or an elementary compact group when $G$ is an elementary compact group.

Condition (b) of the following theorem is similar to property $(P, Q)_{n}$ of Wilder [7, p. 193].

(3.5) THEOREM. If the coefficient group is elementary, then the following are equivalent for a compact space $X$ :

(a) $X$ is $l c^{n}$;

(b) if $A$ and $B$ are closed subsets of $X$ with $A$ in the interior of $B$, then $H_{j}(A ; B)$ is elementary for $j \leqq n$;

(c) if $A$ is a closed subset of $X$ and $U$ is a closed neighborhood of $A$, then there is a closed neighborhood $V$ of $A$ with $H_{j}(V ; U)=H_{j}(A ; U)$ for all $j \leqq n$.

Proof. To show that (a) implies (b), suppose (a) holds and that $A$ is contained in the interior of $B$ (written $A \subset \subset B$ ). Let $A_{-1}=B$. We may find a sequence $B=A_{-1}, A_{0}, \cdots, A_{n}=A$ such that $A_{i+1} \subset \subset A_{i}$. Let $\alpha_{-1}$ be a closed covering of $A_{-1}$. There exists a closed covering $\beta$ of $A_{-1}$ which star-refines $\alpha_{-1}$. Since $A_{0}$ is in the interior of $A_{-1}$ and $X$ is $l c^{n}$, there is a closed covering $\alpha_{0}$ of $A_{0}$ with $\alpha_{0}{ }^{n}>\beta$. By (2.2), $\alpha_{0}^{n} \gg \alpha_{-1}$. Similarly there exist closed coverings $\alpha_{i}$ of $A_{i}$ with $\alpha_{-1} \ll^{n} \alpha_{0} \ll^{n} \ldots \ll^{n} \alpha_{n}$. According to $(2.3 \mathrm{i})$, the kernel $K$ of $\pi_{\alpha_{n}}: H_{j}(A) \rightarrow H_{j}\left(\alpha_{n}\right)$ is contained in the kernel $K^{\prime}$ of $I_{A B}: H_{j}(A) \rightarrow H_{j}(B)$ for $j \leqq n$. Now $H_{j}\left(\alpha_{n}\right)$ is elementary, together with its subgroups and factor groups. Hence $H_{j}(A) / K \approx$ image $\pi_{\alpha_{n}}$ is elementary. Then

$$
H_{j}(A) / K^{\prime} \approx\left(H_{j}(A) / K\right) /\left(K^{\prime} / K\right)
$$

is elementary, being a factor group of an elementary group. But $H_{j}(A ; B)$ $\approx H_{j}(A) / K^{\prime}$; hence (a) implies (b).

Suppose now that (b) holds and that $A \subset \subset U$. For each closed $V$ with $A \subset \subset V \subset U$ let $K(V)=\cap H_{j}\left(V^{\prime} ; V\right)$ where the intersection is taken over all $V^{\prime}$ with $A \subset \subset V^{\prime} \subset \subset V$. Since each $H_{j}\left(V^{\prime} ; V\right)$ is elementary, there is a $V^{\prime}$ with $K(V)=H_{j}\left(V^{\prime} ; V\right)$. We note that $I_{V U}$ maps $K(V)$ onto $K(U)$. For if $x \in K(U)$, then $x \in H_{j}\left(V^{\prime} ; U\right)$ and there is a $y \in H_{j}\left(V^{\prime}\right)$ with $I_{V^{\prime} U}(y)=x$. Then $I_{V U}\left(I_{V^{\prime} V}(y)\right)=x$, and $I_{V^{\prime} V}(y) \in K(V)$. Hence the $K(V)$, together with the $I_{V^{\prime} V}$, constitute an inverse mapping system of elementary groups, and $I_{V U}$ maps $K(V)$ onto $K(U)$. Hence, given $x \in K(U)$, there is a function assigning to each $V$ an $x(V) \in K(V)$ with $x(U)=x$ and $I_{V^{\prime} V}\left(x\left(V^{\prime}\right)\right)=x(V)$. Hence there

(2) An elementary compact group is defined to the direct sum of a finite number of groups, each of which is the reals mod 1 or a finite cyclic group. 
is a $y \in H_{j}(\cap V)=H_{j}(A)$ with $I_{A V}(y)=x(V)$. In particular, $I_{A U}(y)=x$. Then $H_{j}(A ; U)=\cap H_{j}(V ; U)$. But each $H_{j}(V ; U)$ is elementary, so that for some $V, H_{j}(A ; U)=H_{j}(V ; U)$. It follows that (b) implies (c).

It is easy to see that (c) implies (a), by taking for $A$ an arbitrary onepoint set.

(3.6) VIETORIS MAPPING THEOREM. Suppose that $f$ is a continuous map of a compact space $X$ onto a compact space $Y$ such that $H_{j}\left(f^{-1}(y)\right)=0$ for all $j \leqq n$ and $y \in Y$, where the coefficient group is elementary. Then $f_{*}: H_{j}(X) \rightarrow H_{j}(Y)$ is an isomorphism onto for $j \leqq n$, and is onto for $j=n+1$.

Proof. Case I: $X$ is $l_{c^{n+1}}$. Let $\beta_{-1}$ be an arbitrary closed covering of $Y$. Suppose that $\gamma$ is a star-refinement of $\beta_{-1}$; then $f^{-1}(\gamma)$ is a star-refinement of $f^{-1}\left(\beta_{-1}\right)$. Suppose $y \in Y$. Then $y$ is interior to some element $C$ of $\gamma$, and $f^{-1}(y)$ is interior to $f^{-1}(C)$. Since $X$ is $l c^{n}$ and $H_{j}\left(f^{-1}(y)\right)=0$ for $j \leqq n$, there exists by (3.5) a $V$ with $f^{-1}(y) \subset \subset V \subset f^{-1}(C)$ and $H_{j}\left(V ; f^{-1}(C)\right)=0, j \leqq n$. There exists a closed neighborhood $B_{y}$ of $y$ with $f^{-1}\left(B_{y}\right) \subset V$. Let $\beta_{0}$ be a finite subcollection of $\left\{B_{y}\right\}$ whose interiors cover $Y$. Then $f^{-1}\left(\beta_{0}\right)^{n}>f^{-1}(\gamma)$ and $f^{-1}(\gamma)$ star-refines $f^{-1}\left(\beta_{-1}\right)$; by $(2.2), f^{-1}\left(\beta_{0}\right)^{n} \gg f^{-1}\left(\beta_{-1}\right)$. In a similar manner we obtain a sequence $\beta_{-1}<\beta_{0}<\ldots<\beta_{2 n}$ of closed coverings of $Y$ such that if $\alpha_{i}=f^{-1}\left(\beta_{i}\right)$ then $\alpha_{-1} \ll^{n} \ldots \ll^{n} \alpha_{2 n}$. Then by (2.7) $\alpha_{n}, \alpha_{2 n}$ determine $H_{j}(X)$, $j \leqq n$, and partially determine $H_{n+1}(X)$. Since $\beta_{n}$ can be made arbitrarily small, for every sufficiently small covering $\beta$ of $Y$ there exists a refinement $\beta^{\prime}$ of $\beta$ such that the coverings $\alpha=f^{-1}(\beta), \alpha^{\prime}=f^{-1}\left(\beta^{\prime}\right)$ determine $H_{j}(X), j \leqq n$, and partially determine $H_{n+1}(X)$.

Consider the diagram

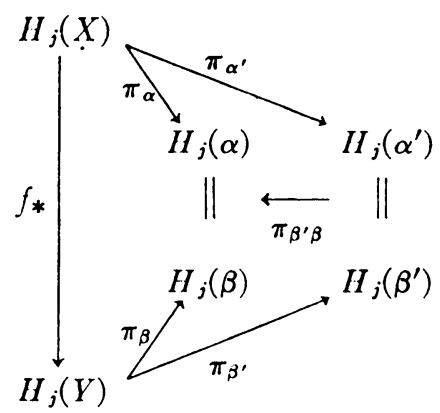

where $\alpha, \beta, \alpha^{\prime}, \beta^{\prime}$ are as above. There is commutativity: $\pi_{\beta} f_{*}=\pi_{\alpha}, \pi_{\beta}, f_{*}=\pi_{\alpha^{\prime}}$, etc.

Note first that $f_{*}$ is an isomorphism into for $j \leqq n$. For $\pi_{\alpha}=\pi_{\beta} f_{*}$ and $\pi_{\alpha}$ is an isomorphism into; hence $f_{*}$ is an isomorphism into.

By (3.3), $H_{j}(X)$ is elementary for $j \leqq n+1$; hence $K=$ image $f_{*}$ is elementary. Moreover, image $\pi_{\beta} f_{*}=$ image $\pi_{\beta}$ for $j \leqq n+1$. For if $y \in H_{j}(Y)$ then 


$$
\pi_{\beta}(y)=\pi_{\beta^{\prime} \beta}\left(\pi_{\beta^{\prime}}(y)\right)=\pi_{\alpha^{\prime} \alpha}\left(\pi_{\beta^{\prime}}(y)\right) .
$$

Since $\alpha, \alpha^{\prime}$ partially determine $H_{j}(X)$, there is an $x \in H_{j}(X)$ with $\pi_{\alpha}(x)$ $=\pi_{\beta}(y)$. Then $\pi_{\beta} f_{*}(x)=\pi_{\beta}(y)$. Hence

$$
\pi_{\beta}(K)=\pi_{\beta}\left(H_{j}(Y)\right) \quad \text { for all sufficiently small } \beta .
$$

Suppose now that $y \in H_{j}(Y)$. $K-y$ denotes the set $\{k-y: k \in K\}$. For each $\beta$ there is a $k \in K$ with $\pi_{\beta}(k)=\pi_{\beta}(y)$, so that $k-y \in L_{\beta}=\left(\right.$ kernel $\left.\pi_{\beta}\right)$ $\cap(K-y)$. Now $K-y$ is a translate of $K$, which is elementary. The $L_{\beta}$ are nonempty and decrease with $\beta$. Hence $\bigcap_{\beta} L_{\beta} \neq \varnothing$. If $z \in \bigcap_{\beta} L_{\beta}$, then $\pi_{\beta}(z)=0$ for all $\beta$, so that $z=0$. Hence $0 \in K-y$ and $y \in K$. Hence $f_{*}$ maps $H_{j}(X)$ onto $H_{j}(Y), j \leqq n+1$.

Case II : $X$ a compact space. We may consider $X$ as embedded in an $l c^{n+1}$ compact space $X^{\prime}$ (for example, $X^{\prime}$ a product of intervals). The map $f$ generates a decomposition of $X$ whose elements are the sets $f^{-1}(y), y \in Y$. Extend this decomposition to a decomposition of $X^{\prime}$ by admitting as elements the one-point sets of $X^{\prime}-X$. This upper semi-continuous decomposition of $X^{\prime}$ has a decomposition space $Y^{\prime}$ and a decomposition map $F: X^{\prime} \rightarrow Y^{\prime}$. We may identify $Y$ with $F(X)$ and $f$ with $F \mid X$. We have the diagram

$$
\begin{aligned}
& H_{j+1}\left(X^{\prime}\right) \rightarrow H_{j+1}\left(X^{\prime}, X\right) \rightarrow H_{j}(X) \rightarrow H_{j}\left(X^{\prime}\right) \rightarrow H_{j}\left(X^{\prime}, X\right) \\
& \downarrow F_{*_{1}} \quad \downarrow F_{*}^{\prime} \quad \downarrow f_{*} \quad \downarrow F_{*_{2}} \quad \downarrow F_{*}^{\prime} \\
& H_{j+1}\left(Y^{\prime}\right) \rightarrow H_{j+1}\left(Y^{\prime}, Y\right) \rightarrow H_{j}(Y) \rightarrow H_{j}\left(Y^{\prime}\right) \rightarrow H_{j}\left(Y^{\prime}, Y\right)
\end{aligned}
$$

where the downward homomorphisms are induced by $F$. Now both the $F_{*}^{\prime}$ are isomorphisms onto, since $F$ maps $X^{\prime}-X$ homeomorphically onto $Y^{\prime}-Y$. For $j \leqq n, F_{* 2}$ is an isomorphism onto by Case I, and $F_{* 1}$ is onto. By the fivelemma of Eilenberg-Steenrod [2, p. 16], $f_{*}$ is an isomorphism onto. For $j=n+1, F_{* 2}$ is onto; by the five-lemma, $f_{*}$ is then onto. The theorem follows.

4. Regular convergence. In this section, we prove some theorems concerning regular convergence. For the known facts where the coefficient group is a field, see [5].

(4.1) Definition. A sequence $\left(A_{i}\right)$ of closed subsets of a compact space $X$ is said to converge n-regularly to the subset $A$ of $X$ if and only if $\left(A_{i}\right)$ converges to $A$, and given $x \in A$ and a closed neighborhood $U$ of $x$ there exist a closed neighborhood $V$ of $x$ and a positive integer $N$ such that $H_{j}\left(V \cap A_{i}\right.$; $\left.U \cap A_{i}\right)=0$ for $j \leqq n$ and $i \geqq N$.

If $\beta=\left(B^{1}, \cdots, B^{r}\right)$ is a covering of $X$ and $A \subset X$ then $\beta \cap A$ denotes the covering $\left(B^{1} \cap A, \cdots, B^{r} \cap A\right)$ of $A$.

(4.2) If the sequence $\left(A_{i}\right)$ converges n-regularly to $A$, then given a closed covering $\alpha$ of $X$ there exist a closed covering $\beta$ of $X$ and a positive integer $N$ such that $\alpha \cap A_{i} \ll n \beta \cap A_{i}$ for all $i \geqq N$.

Proof. Let $\gamma$ be a closed covering of $X$ such that $\gamma$ star-refines $\alpha$. For each 
$x \in X$ there is a $C$ in $\gamma$ with $x$ in its interior. Hence there is a closed neighborhood $B_{x}$ of $x$ and a positive integer $N_{x}$ such that $H_{j}\left(B_{x} \cap A_{i} ; C \cap A_{i}\right)=0$ for $j \leqq n$ and $i \geqq N_{x}$. Let $\beta$ be a finite subcollection of $B_{x}$ whose interiors cover $X$. Then $\beta \cap A_{i}{ }^{n}>\gamma \cap A_{i}$ for $i$ sufficiently large. Since $\gamma \cap A_{i}$ star-refines $\alpha \cap A_{i}$, then $\beta \cap A_{i}{ }^{n} \gg \alpha \cap A_{i}$ for $i$ sufficiently large.

(4.3) If $\left(A_{i}\right)$ converges $n$-regularly to $A$, then for each sufficiently small closed covering $\alpha$ of $X$, there exists a closed covering $\beta>\alpha$ and a positive integer $N$ such that $\alpha \cap A_{i}, \beta \cap A_{i}$ determine $H_{j}\left(A_{i}\right)$ for $j \leqq n$ and $i \geqq N$ and partially determine $H_{n+1}\left(A_{i}\right)$ for $i \geqq N$.

Proof. The proof follows easily from (4.2) and (2.7).

(4.4) Definition. Suppose that $A$ is a closed subset of the compact space $X$, and that $\alpha$ is a closed covering of $X$. We say that $\alpha$ is in general position relative to $A$ if and only if whenever $A^{i_{0}}, \cdots, A^{i_{q}}$ are elements of $\alpha$ with $A^{i_{0}} \cap \cdots \cap A^{i_{q}} \cap A \neq \varnothing$ then int $A^{i_{0}} \cap \cdots \cap$ int $A^{i_{q}} \cap A \neq \varnothing$.

(4.5) If $A$ is a closed subset of the compact space $X$ and $\alpha$ is a closed covering of $X$, there is a refinement $\beta$ of $\alpha$ in general position relative to $A$.

Proof. Suppose $\alpha=\left(A^{1}, \cdots, A^{r}\right)$. For each $i_{0}, \cdots, i_{q}$, select a point $x_{i_{0}}, \ldots, i_{q}$ of int $A^{i_{0}} \cap \cdots \cap \operatorname{int} A^{i_{q}} \cap A$, if such a point exists. Let $\beta$ $=\left(B^{1}, \cdots, B^{r}\right)$ be a closed covering of $X$ such that $B^{i}$ is in the interior of $A^{i}$ and such that $B^{i}$ contains (in its interior) all the $x_{i_{0}}, \ldots, i q$ which belong to int $A^{i}$. Then $\beta$ is the desired covering.

It will be noted that if $\left(A^{i}\right)$ converges to $A$, and $\alpha$ is general position relative to $A$, then the nerves of $\alpha \cap A$ and $\alpha \cap A_{i}$ coincide for $i$ sufficiently large.

(4.6) Theorem. Suppose that the sequence $\left(A_{i}\right)$ of closed subsets of the compact space $X$ converges to $A$, and that for each sufficiently small closed covering $\alpha$ of $X$ there exists a closed covering $\beta$ of $X$ and a positive integer $N$ such that $\alpha \cap A_{i}, \beta \cap A_{i}$ determine $H_{n}\left(A_{i}\right)$ for $i \geqq N$. Then for each $\alpha$, there is a $\beta$ such that $\alpha \cap A, \beta \cap A$ determine $H_{n}(A)$.

Proof. We consider closed coverings $\alpha<\beta<\gamma$ which are in general position relative to $A$. We use the diagram

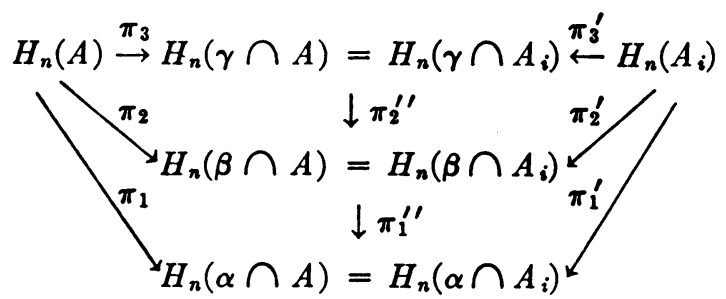

where all the $\pi$ 's are projections and where $i$ is always large enough so that the indicated equalities hold.

Suppose that $x \in H_{n}(A), x \neq 0$, and $\pi_{1}(x)=0$. Suppose $\alpha \cap A_{i}, \beta \cap A_{i}$ deter- 
mine $H_{n}\left(A_{i}\right), i \geqq N$, that $\pi_{2}(x) \neq 0$, and that $\beta \cap A_{i}, \gamma \cap A_{i}$ determine $H_{n}\left(A_{i}\right)$, $i \geqq N$. Since $\pi_{3}(x)=\pi_{2}^{\prime \prime} \pi_{2}(x)$, there is a $y \in H_{n}\left(A_{i}\right)$ with $\pi_{2}^{\prime}(y)=\pi_{2}(x)$. Then $y \neq 0$ since $\pi_{2}^{\prime}(y) \neq 0$. Then also $\pi_{1}^{\prime}(y) \neq 0$, since $\alpha \cap A_{i}, \beta \cap A_{i}$ determine $H_{n}\left(A_{i}\right)$. But $\pi_{1}^{\prime}(y)=\pi_{1}(x)$. This is a contradiction. Hence $\pi_{1}$ is an isomorphism into.

Suppose that $\alpha \cap A_{i}, \beta \cap A_{i}$ determine $H_{n}\left(A_{i}\right), i \geqq N$. We show that image $\pi_{1}=$ image $\pi_{1}^{\prime \prime}$; we know that image $\pi_{1}$ Cimage $\pi_{1}^{\prime \prime}$. Let $y \in H_{n}(\beta \cap A)$. There is an $x_{i} \in H_{n}\left(A_{i}\right)$ with $\pi_{1}^{\prime}(x)=\pi_{1}^{\prime \prime}(y)$. For each closed covering $\gamma$ of $X, \gamma$ in general position relative to $A$, with $\gamma>\beta, \pi_{1}^{\prime \prime} \pi_{2}^{\prime \prime}\left(\pi_{3}^{\prime}\left(x_{i}\right)\right)=\pi_{1}^{\prime}\left(x_{i}\right)=\pi_{1}^{\prime \prime}(y)$. Hence for each such $\gamma$ there is a $z_{\gamma}\left(=\pi_{3}^{\prime}\left(x_{i}\right)\right)$ in $H_{n}(\gamma \cap A)$ with $\pi_{1}{ }^{\prime \prime} \pi_{2}^{\prime \prime}\left(z_{\gamma}\right)$ $=\pi_{1}^{\prime \prime}(y)$. Hence there is an $x \in H_{n}(A)$ whose $(\alpha \cap A)$-coordinate is $\pi_{1}^{\prime}(y)$. Then $\pi_{1}(x)=\pi_{1}^{\prime \prime}(y)$. Hence if $\alpha \cap A_{i}, \beta \cap A_{i}$ determine $H_{n}\left(A_{i}\right), i \geqq N$, and $\alpha, \beta$ are in general position relative to $A$ then $\alpha \cap A, \beta \cap A$ determine $H_{n}(A)$.

(4.7) Theorem. Suppose that the sequence $\left(A_{i}\right)$ of closed subsets of the compact space $X$ converges n-regularly to the subset $A$. Then $H_{n}(A) \approx H_{n}\left(A_{i}\right)$ for $i$ sufficiently large.

Proof. There exist, by (4.5) and (4.3), closed coverings $\alpha, \beta$ of $X$, in general position relative to $A$, such that $\alpha \cap A_{i}, \beta \cap A_{i}$ determine $H_{n}\left(A_{i}\right)$. By (4.6), $\alpha \cap A, \beta \cap A$ determine $H_{n}(A)$. Hence $H_{n}\left(A_{i}\right)$ and $H_{n}(A)$, for $i$ sufficiently large, are isomorphic to the image of the projection $\pi: H_{n}(\beta \cap A) \rightarrow H_{n}(\alpha \cap A)$, and are isomorphic to each other.

5. The Kelley-Pitcher theory for simplicial pairs. In $\S \S 5$ and 6 we continue an investigation of Kelley and Pitcher [3, pp. 703-706] seeking relationships between the groups of a space, the groups of the nerve of a covering of the space, and the groups of intersections of elements of the covering. The treatment is self-contained; the portion of this section through (5.5) is due to Kelley-Pitcher.

By a simplicial pair we mean a pair $(X, \alpha)$ consisting of a finite simplicial complex $X$ and a covering $\alpha=\left(A^{1}, \cdots, A^{r}\right)$ by subcomplexes. $X_{\alpha}$ will denote the nerve of $\alpha$. However, $C_{p}(\alpha)$ and $H_{p}(\alpha)$ will indicate the chain group and homology group of the nerve $X_{\alpha}$. If a simplex of $X_{\alpha}$ is of the form $T_{q}$ $=\left(\tilde{A}^{i_{0}} \cdots \tilde{A}^{i_{q}}\right)$ then define $\cap T_{q}=A^{i_{0}} \cap \cdots \cap A^{i_{q}}$. Consider $X_{\alpha}$ as having a (-1)-dimensional simplex $T_{-1}$ with $\cap T_{-1}=X$.

If $S_{p}$ is a $p$-simplex of $X$ let $\wedge S_{p}$ denote the subcomplex of $X_{\alpha}$ consisting of all $T_{q}$ with $S_{p} \subset \cap T_{q}$. If $A^{i_{0}}, \cdots, A^{i_{q}}$ are all the elements of $\alpha$ which contain $S_{p}, p \geqq 0$, then $\Lambda S_{p}$ consists of all faces of the simplex $\left(\tilde{A}^{i_{0}} \ldots \tilde{A}^{i_{q}}\right)$. If $S_{-1}$ is the (-1)-dimensional simplex of $X$, then $\Lambda S_{-1}=X_{\alpha}$. The boundary operator will be denoted by $\partial$.

Denote by $C_{p, q}(\alpha)$ the set of all linear forms $\sum g S_{p} \cdot T_{q}$ where $g \in(S)$ and $S_{p}, T_{q}$ are oriented simplices of $X, X_{\alpha}$ respectively with $S_{p} \subset \cap T_{q}$. Agree that $\mathfrak{g} S_{p} \cdot\left(-T_{q}\right)=\mathfrak{g}\left(-S_{p}\right) \cdot T_{q}=(-\mathfrak{g}) S_{p} \cdot T_{q}$ and that forms are to be added as 
usual. If $A_{p}=\sum g_{i} S_{p}^{t}$ is a $p$-chain of $\cap T_{q}$, define $A_{p} \cdot T_{q}=\sum g_{i} S_{p}^{t} \cdot T_{q}$. If $B_{q}=\sum g_{i} T_{q}^{i}$ is a $q$-chain of $\Lambda S_{p}$, define $S_{p} \cdot B_{q}=\sum g_{i} S_{p} \cdot T_{q}^{i}$.

The group $C_{p,-1}(\alpha)$ will be identified with the chain group $C_{p}(X)$ under the identification $A_{p} \cdot T_{-1} \leftrightarrow A_{p}$. The group $C_{-1, q}(\alpha)$ will be identified with the chain group $C_{q}(\alpha)$ under the identification $S_{-1} \cdot B_{q} \leftrightarrow B_{q}$.

$A$ homomorphism $\Delta: C_{p, q} \rightarrow C_{p-1, q}$ is defined by $\Delta\left(A_{p} \cdot S_{q}\right)=\left(\partial A_{p}\right) \cdot S_{q}$ and linearity. $A$ homomorphism $D: C_{p, q} \rightarrow C_{p, q-1}$ is defined by $D\left(S_{p} \cdot B_{q}\right)=S_{p} \cdot\left(\partial B_{q}\right)$ and linearity.

(5.1) If $p \geqq 0$ the sequence

$$
\cdots \rightarrow C_{p, q+1} \stackrel{D}{\rightarrow} C_{p, q} \stackrel{D}{\rightarrow} C_{p, q-1} \rightarrow \cdots
$$

is exact. If $p=-1$, the sequence coincides with

$$
\cdots \rightarrow C_{q+1}(\alpha) \stackrel{\partial}{\rightarrow} C_{q}(\alpha) \stackrel{\partial}{\rightarrow} C_{q-1}(\alpha) \rightarrow \cdots .
$$

Proof. It is clear that $D D=0$ since $\partial \partial=0$. Suppose $p \geqq 0$ and that $D\left(\sum S_{p}^{t} \cdot B_{q}^{i}\right)=\sum S_{p}^{i} \cdot\left(\partial B_{q}^{i}\right)=0$, where the $S_{p}^{i}$ form a basis for $C_{p}(X)$. Then $\partial B_{q}^{i}=0$. Since $B_{q}^{i}$ is on the simplex $\Lambda S_{p}^{t}$, then $B_{q}^{i}=\partial C_{q}^{i}$ for some $C_{q}^{t}$ in $\Lambda S_{p}^{t}$. Then $D\left(\sum S_{p}^{\imath} \cdot C_{q}^{i}\right)=\sum S_{p}^{i} \cdot B_{q}^{i}$, and the sequence is exact for $p \geqq 0$. The statement for $p=-1$ can be easily seen.

(5.2) The sequence

$$
\cdots \rightarrow C_{p+1, q} \stackrel{\Delta}{\rightarrow} C_{p . q} \stackrel{\Delta}{\rightarrow} \ldots
$$

has $\Delta \Delta=0$. The homology group $H_{p, q}(\alpha)$ of the sequence corresponding to the indices $p, q$ is isomorphic to the direct sum $\sum H_{p}\left(\cap T_{q}\right)$, where the summation is extended over all $q$-simplices $T_{q}=\left(\tilde{A}^{i_{0}} \ldots \widetilde{A}^{i_{q}}\right)$ with $i_{0}<\ldots<i_{q}$.

Proof. It is clear that $\Delta \Delta=0$. If $q=-1, C_{p,-1}=C_{p}(X)$ and $\Delta=\partial$ on $C_{p}(X)$. Hence the homology group, if $q=-1$, is $H_{p}(X)=H_{p,-1}(\alpha)$. Suppose $q \geqq 0$. Elements $z$ of $C_{p, q}$ are uniquely represented as $z=\sum A_{p}^{i} \cdot T_{a}^{i}$, where the $T_{a}^{i}$ are the oriented $q$-simplices of the form $\left(\widetilde{A}^{i_{0}} \ldots \widetilde{A}^{i_{q}}\right)$ with $i_{0}<\ldots<i_{q}$ and where $A_{p}^{i} \in C_{p}\left(\cap T_{q}^{i}\right)$. This representation sets up an isomorphism of $C_{p, q}$ with $\sum C_{p}\left(\cap S_{q}\right)$ and the statement follows easily.

Define $E_{p, q}$ to be the kernel of $D: C_{p, q} \rightarrow C_{p, q-1}$. For $p \geqq 0$, it follows from (5.1) that $E_{p, q}$ is the image of $D: C_{p, q+1} \rightarrow C_{p, q}$. We have the sequence

$$
\cdots \rightarrow E_{p+1, q} \stackrel{\Delta}{\rightarrow} E_{p, q} \stackrel{\Delta}{\rightarrow} \cdots .
$$

Define $K_{p, q}$ to be the homology group of this sequence corresponding to indices $p, q$.

(5.3) We have $K_{p,-1}=H_{p}(X)$.

Proof. $K_{p,-1}$ is the homology group of

$$
\cdots \rightarrow E_{p+1,-1} \stackrel{\Delta}{\rightarrow} E_{p,-1} \stackrel{\Delta}{\rightarrow} E_{p-1,-1} \rightarrow \cdots .
$$


Now $D: C_{p,-1} \rightarrow C_{p,-2}=0$ so that $E_{p,-1}=C_{p,-1}=C_{p}(X)$. Moreover, $\Delta=\partial$ on $C_{p}(X)$. The result follows.

(5.4) We have $K_{-1, q}=H_{q}(\alpha)$.

Proof. We have $C_{-1, q}=C_{q}(\alpha)$. Also $D: C_{-1, q} \rightarrow C_{-1, q-1}$ coincides with $\partial: C_{q}(\alpha) \rightarrow C_{q-1}(\alpha)$. Hence $E_{-1, q}$ is the cycle group $Z_{q}(\alpha)$. Now $E_{0, q}$, is the image of $D: C_{0, q+1} \rightarrow C_{0, q}$. Hence the image of $\Delta: E_{0, q} \rightarrow E_{-1, q}$ is also the image of $\Delta D: C_{0, q+1} \rightarrow C_{-1, q}$. But $\Delta D\left(\sum A_{0}^{i} \cdot T_{q+1}^{i}\right)=\sum\left(\partial A_{0}^{i}\right) \cdot\left(\partial T_{q+1}^{i}\right)$ where $\partial A_{0}^{i}$ is of the form $\mathfrak{g}_{i} S_{-1}$ with $\mathfrak{g}_{i}$ the coefficient sum of $A_{0}^{i}$. It follows that the image of $\Delta: E_{0, q} \rightarrow E_{-1, q}$ is the bounding cycle group $B_{q}(\alpha)$. The result follows.

For each $p \geqq 0$, the sequence

$$
0 \rightarrow E_{p, q} \stackrel{l^{\prime}}{\rightarrow} C_{p, q} \stackrel{D}{\rightarrow} E_{p, q-1} \rightarrow 0
$$

is exact, where $l^{\prime}$ is inclusion. If $p=-1$ the sequence is exact except that $D$ may not be onto. Taking homology groups and using a well-known method of constructing exact sequences $[3$, p. 687] we get the exact sequence

$$
\cdots \rightarrow K_{p, q} \stackrel{l}{\rightarrow} H_{p, q} \stackrel{m}{\rightarrow} K_{p, q-1} \stackrel{\eta^{(p)}}{\rightarrow} K_{p-1, q} \rightarrow \cdots
$$

$\rightarrow K_{0, q-1} \rightarrow K_{-1, q} \rightarrow H_{-1, q}$, where $l$ is induced by inclusion $l^{\prime}, m$ by $D$, and $\eta^{(p)}$ by the boundary operation. Since $H_{-1, q}=0$, we get

(5.5) The sequence

$$
\cdots \rightarrow K_{p, q} \stackrel{l}{\rightarrow} H_{p, q} \stackrel{m}{\rightarrow} K_{p, q-1} \stackrel{\eta^{(p)}}{\rightarrow} K_{p-1, q} \rightarrow \cdots \rightarrow K_{0, q-1} \rightarrow K_{-1, p} \rightarrow 0
$$

is exact.

The map $\eta^{(p)}: K_{p, q-1} \rightarrow K_{p-1, q}, p \geqq 0$, is of particular interest. Suppose $z$ represents an element $x$ of $K_{p, q-1}$. Then $z=D\left(z^{\prime}\right)$ where $z^{\prime} \in C_{p, q}$. Then $\Delta z^{\prime}$ represents $\eta^{(p)}(x)$.

$$
\text { In } \begin{aligned}
H_{p}(X) & =K_{p,-1} \stackrel{\eta^{(p)}}{\rightarrow} K_{p-1,0} \stackrel{\eta^{(p-1)}}{\longrightarrow} K_{p-1,2} \rightarrow \cdots \rightarrow K_{0, p-1} \stackrel{\eta^{(0)}}{\longrightarrow} K_{-1, p} \\
& =H_{p}(\alpha), \text { define } \eta=\eta^{(0)} \cdots \eta^{(p-1)} \eta^{(p)} .
\end{aligned}
$$

(5.6) Theorem. Suppose $(X, \alpha)$ is a simplicial pair such that if $S$ is a simplex of $X$ there is a vertex $v$ of $S$ such that if $v \in A^{i}$ then $S \subset A^{i}$. Any function $f$ assigning to each vertex $v$ of $X$ a vertex $\tilde{A}^{i}$ of $X_{\alpha}$ with $v \in A^{i}$ is a simplicial map of $X$ into $X_{\alpha}$. The homomorphism $f_{*}: H_{p}(X) \rightarrow H_{p}(\alpha)$ is given by $f_{*}=\epsilon \eta$, where $\epsilon=(-1)^{p(p+1) / 2}$.

Proof. For a vertex $v$ of $X, \Lambda_{v}$ denotes the simplex of $X_{\alpha}$ whose vertices are those $\tilde{A}^{i}$ with $v \in A^{i}$. Suppose $v \sim v^{\prime}$ if and only if $\Lambda v=\Lambda v^{\prime}$. This is an equivalence relation. Suppose the elements of each equivalence class are simply ordered by an ordering $>$. Suppose also that $v>v^{\prime}$ whenever $\Lambda v$ contains $\Lambda v^{\prime}$ as a proper face. We have then a partial ordering $>$ on the vertices of $X$ 
such that if $v>v^{\prime}$ then $\Lambda v \supset \Lambda v^{\prime}$, if $\Lambda v=\Lambda v^{\prime}$ either $v>v^{\prime}$ or $v^{\prime}>v$, if $\Lambda v$ contains $\Lambda v^{\prime}$ properly then $v>v^{\prime}$.

Suppose $S$ is a simplex of $X$. There is a vertex $v$ such that if $v^{\prime}$ is any other vertex of $S$ then $\Lambda v \subset \Lambda v^{\prime}$. It follows that there is a vertex $v_{0}$ of $S$ (with $\Lambda v_{0}=\Lambda v$ ) such that $v_{0}<v^{\prime}$ for all vertices $v^{\prime}$ of $S$. It then follows that the vertices of $S$ may be ordered uniquely as $v_{0}<v_{1}<\cdots<v_{p}$. In this proof, all oriented simplices $S=\left(v_{0} \cdots v_{p}\right)$ will have $v_{0}<\cdots<v_{p}$. If $v_{0} \in A^{i}$ then $S \subset A^{i}$. Also if $v_{i} \in A^{i}$ then $v_{p} \in A^{i}$.

Consider $f$ as in the statement of the theorem. If $S=\left(v_{0} \cdots v_{p}\right)$ then $f\left(v_{j}\right)$ is a vertex $\tilde{A}^{i_{i}}$ with $v_{j} \in A^{i_{0}}$. Then $v_{p} \in A^{i_{0}} \cap \cdots \cap A^{i_{p}}$ and $f$ is simplicial.

A vertex of $X_{\alpha}$ will be denoted by a symbol of the form $w_{j}$. Define a homomorphism $\mathrm{J}: C_{p, q} \rightarrow C_{p, q+1}, p \geqq 0$, by

$$
g\left(v_{0} \cdots v_{p}\right) \cdot\left(w_{0} \cdots w_{q}\right) \rightarrow g\left(v_{0} \cdots v_{p}\right) \cdot\left(f\left(v_{0}\right) w_{0} \cdots w_{q}\right)
$$

and linearity. If $S=\left(v_{0} \cdots v_{p}\right)$ then $\left(w_{0} \cdots w_{q}\right) \subset \Lambda S$ and $f\left(v_{0}\right) \epsilon \wedge S$. Since $\Lambda S$ is a simplex, the definition has meaning.

It may be verified that $D J+\Im D=$ identity. Hence if $D(z)=0$ then $D(T(z))$ $=z$. Hence $(*)$ if $z$ represents an element $x$ of $K_{p, q}, p \geqq 0$, then $\Delta T(z)$ represents $\eta^{(p)}(x)$ in $K_{p-1, q+1}$.

Let $z=\sum g_{i}\left(v_{0 i} \cdots v_{p i}\right)$ represent an element $x$ of $K_{p,-1}=H_{p}(X)$. By $\left(^{*}\right)$, $\eta^{(p)}(x)$ in $K_{p-1,0}$ is represented by

$$
\begin{aligned}
& \sum g_{i} \partial\left(v_{0 i} \cdots v_{p i}\right) \cdot f\left(v_{0 i}\right) \\
&=\sum g_{i}\left(v_{1 i} \cdots v_{p i}\right) \cdot f\left(v_{0 i}\right)+\sum_{j>0}(-1)^{i} g_{i}\left(v_{0 i} \cdots \hat{v}_{j i} \cdots v_{p i}\right) \cdot f\left(v_{0 i}\right) .
\end{aligned}
$$

By $\left(^{*}\right)$ again, $\eta^{(p-1)} \eta^{(p)}(x)$ in $K_{p-1,2}$ is represented by

$\sum g_{i} \partial\left(v_{1 i} \cdots v_{p i}\right) \cdot\left(f\left(v_{1 i}\right) f\left(v_{0 i}\right)\right)$

$$
\begin{aligned}
= & \sum g_{i}\left(v_{2 i} \cdots v_{p i}\right) \cdot\left(f\left(v_{1 i}\right) f\left(v_{0 i}\right)\right) \\
& +\sum_{j>1}(-1)^{j-1} g_{i}\left(v_{1 i} \cdots \hat{v}_{j i} \cdots v_{p i}\right) \quad\left(f\left(v_{1 i}\right) f\left(v_{0 i}\right)\right) .
\end{aligned}
$$

Continuing, the element $\eta^{(1)} \cdots \eta^{(p)}(x)$ in $K_{0, p-1}$ is represented by

$$
\begin{aligned}
& \sum g_{i} \partial\left(v_{p-1 i} v_{p i}\right)\left(f\left(v_{p-1 i}\right) \cdots f\left(v_{0 i}\right)\right) \\
&=\sum g_{i} v_{p i} \cdot\left(f\left(v_{p-1 i}\right) \cdots f\left(v_{0 i}\right)\right)-\sum g_{i} v_{p-1 i} \cdot\left(f\left(v_{p-1 i}\right) \cdots f\left(v_{0 i}\right)\right) .
\end{aligned}
$$

Hence $\eta(x)$ is represented by

$$
\sum \mathfrak{g}_{i} S_{-1} \cdot\left(f\left(v_{p i}\right) \cdots f\left(v_{0 i}\right)\right)=(-1)^{p(p+1) / 2} f(z) .
$$

The theorem follows for $p \geqq 0$. For $p=-1$ the theorem is trivial.

If $(X, \alpha)$ and $(Y, \beta)$ are simplicial pairs then $a$ map $F:(X, \alpha) \rightarrow(Y, \beta)$ is a pair $F=(f, g)$ consisting of a simplicial map $f: X \rightarrow Y$ and a function $g: \alpha \rightarrow \beta$ which assigns to each $A^{i}$ in $\alpha$ an element $g A^{i}=B^{i}$ of $\beta$ with $f A^{i} \mathrm{Cg} A^{i}$. The cor- 
responding simplicial map from $X_{\alpha}$ to $Y_{\beta}$ will also be denoted by $g$. Suppose $S_{p} \subset \cap T_{q}$ where $T_{q}$ is a simplex of $X_{\alpha}$. Then $f\left(S_{p}\right) \subset \cap_{g} T_{q}$. If $A$ is a chain of $X$ then $f A$ will denote its image under $f$. There is the function

$$
\sum A^{i} \cdot T_{q}^{i} \rightarrow \sum f A^{i} \cdot g T_{q}^{i}
$$

of $C_{p, q}(\alpha)$ into $C_{p, q}(\beta)$, which we denote by $F_{c}$. It may be seen that $D F_{c}=F_{c} D$. Hence $F_{c}$ maps $E_{p, q}(\alpha)$ into $E_{p, q}(\beta)$. Moreover, since $\Delta F_{c}=F_{c} \Delta$, there is induced a map $F_{k}: K_{p, q}(\alpha) \rightarrow K_{p, q}(\beta)$. Similarly $F_{c}$ induces a map $F_{h}: H_{p, q}(\alpha)$ $\rightarrow H_{p, q}(\beta)$. The following may be seen.

(5.7) There is commutativity in each rectangle of

$$
\begin{aligned}
& \cdots \rightarrow K_{p, q}(\alpha) \rightarrow H_{p, q}(\alpha) \rightarrow K_{p, q-1}(\alpha) \rightarrow \cdots \\
& \downarrow F_{k} \quad \downarrow F_{h} \quad \downarrow F_{k} \\
& \cdots \rightarrow K_{p, q}(\beta) \rightarrow H_{p, q}(\beta) \rightarrow K_{p, q-1}(\beta) \rightarrow \cdots .
\end{aligned}
$$

Moreover, $F_{k}: K_{p,-1}(\alpha) \rightarrow K_{p,-1}(\beta)$ coincides with $f_{*}: H_{p}(X) \rightarrow H_{p}(Y)$ and $F_{k}: K_{-1, q}(\alpha) \rightarrow K_{-1, q}(\beta)$ with $g_{*}: H_{q}(\alpha) \rightarrow H_{q}(\beta)$.

(5.8) Suppose that $F=(f, g)$ and $F^{\prime}=\left(f^{\prime}, g\right)$ are two maps of $(X, \alpha)$ into $(Y, \beta)$ such that given a simplex $S$ of $A^{i} \in \alpha$ then there is a simplex $S^{\prime}$ of $g A^{i} \in \beta$ such that $f(S) \cup f^{\prime}(S) \subset S^{\prime}$. Then $F_{h}=F_{h}^{\prime}$ and $F_{k}=F_{k}^{\prime}$.

Proof. For a simplex $S$ of $X$ suppose that $S^{\prime}$ is the smallest simplex of $Y$ containing $f(S) \cup f^{\prime}(S)$. If $S \in \cap T_{q}$ then $S^{\prime} \in \bigcap_{g} T_{q}$. Define J: $C_{p . q}(\alpha) \rightarrow C_{p+1, q}(\beta)$, $p \geqq 0$, by

$$
\mathfrak{g}\left(v_{0} \cdots v_{p}\right) \cdot T_{q} \rightarrow\left[\sum(-1){ }^{i} \mathfrak{g}\left(f\left(v_{0}\right) \cdots f\left(v_{i}\right) f^{\prime}\left(v_{i}\right) \cdots f^{\prime}\left(v_{p}\right)\right)\right] \cdot g T_{q}
$$

and linearity. Then $\Delta J+J \Delta=F_{c}^{\prime}-F_{c}$ for $p \geqq 1, \Delta J=F_{c}^{\prime}-F_{c}$ for $p=0$. Moreover, $D J=\Im D$. Hence $\Im: E_{p, q}(\alpha) \rightarrow E_{p+1, q}(\beta), p \geqq 0$. It follows that $F_{h}=F_{h}^{\prime}$ and $F_{k}=F_{k}^{\prime}$ for $p \geqq 0$.

The case $p=-1$ remains. On $C_{-1, p}, F_{c}=F_{c}^{\prime}$. Hence the conclusion follows for $p=-1$.

The following theorem may be easily proved.

(5.9) If

$$
(X, \alpha) \stackrel{F}{\rightarrow}(Y, \beta) \stackrel{F^{\prime}}{\rightarrow}(Z, \gamma)
$$

then $\left(F^{\prime} F\right)_{h}=F_{h}^{\prime} F_{h}$ and $\left(F^{\prime} F\right)_{k}=F_{k}^{\prime} F_{k}$.

6. The Kelley-Pitcher theory of compact pairs. In this section we extend the Kelley-Pitcher theory to pairs $(X, \alpha)$, where $X$ is a compact space and $\alpha=\left(A^{1}, \cdots, A^{r}\right)$ a collection of closed subsets of $X$ which covers $X$. We begin the section by summarizing the results.

For such a compact pair $(X, \alpha)$, define $H_{p, q}(\alpha)=\sum H_{p}\left(\cap T_{q}\right)$, where the summation is over all q-simplices $T_{q}=\left(\tilde{A}^{i_{0}} \cdots \tilde{A}^{i_{q}}\right)$ of $X_{\alpha}$ with $i_{0}<\cdots<i_{q}$. If $T_{q}$ is a simplex as above and if $A_{p} \in H_{p}\left(\cap T_{q}\right)$ then denote by $A_{p} \cdot T_{q}$ the 
element of $H_{p, q}$ whose $T_{q}$-coordinate is $A_{p}$ and all of whose other coordinates are 0 . Agree also that $A_{p} \cdot\left(-T_{q}\right)=\left(-A_{p}\right) \cdot T_{q}$. Then $H_{p, q}$ is the set of linear forms $\sum A_{p}^{i} \cdot T_{q}^{i}$ where $A_{p}^{i} \in H_{p}\left(\cap T_{q}^{i}\right)$.

$A$ map $F$ of a pair $(X, \alpha)$ into a pair $(Y, \beta)$ will be a pair $F=(f, g)$ where $f: X \rightarrow Y$ is continuous and where $g: \alpha \rightarrow \beta$ assigns to each $A^{i}$ in $\alpha$ an element $g A^{i}=B^{i}$ in $\beta$ such that $f A^{i} C_{g} A^{i}$. The simplicial map of $X_{\alpha}$ into $Y_{\beta}$ will also be denoted by $g$. If $T_{q}$ is an unoriented simplex of $X_{\alpha}$ then $f\left(\cap T_{q}\right) \subset \cap g T_{g}$. The map $f$ of $\cap T_{q}$ into $\cap g T_{q}$ is denoted by $f \mid \cap T_{q}$. There is the induced homomorphisms $\left(f \mid \cap T_{q}\right)_{*}: H_{p}\left(\cap T_{q}\right) \rightarrow H_{p}\left(\cap g T_{q}\right)$. A homomorphism $F_{h}: H_{p, q}(\alpha)$ $\rightarrow H_{p . q}(\beta)$ is defined by $A_{p} \cdot T_{q} \rightarrow\left[\left(f \mid \cap T_{q}\right)_{*} A_{p}\right] \cdot g T_{q}$ and linearity.

If

$$
(X, \alpha) \stackrel{F}{\rightarrow}(Y, \beta) \stackrel{F^{\prime}}{\rightarrow}(Z, \gamma)
$$

where $F=(f, g)$ and $F^{\prime}=\left(f^{\prime}, g^{\prime}\right)$ then the composition $F^{\prime} F:(X, \alpha) \rightarrow(Z, \gamma)$ is defined to be $\left(f^{\prime} f, g^{\prime} g\right)$.

We define for each $(X, \alpha)$ a group $K_{p, q}(\alpha)$; a map $F:(X, \alpha) \rightarrow(Y, \beta)$ will induce a homomorphism $F_{k}: K_{p, q}(\alpha) \rightarrow K_{p, q}(\beta)$. There will also be homomorphisms

$$
K_{p, q} \stackrel{l}{\rightarrow} H_{p, q} \stackrel{m}{\rightarrow} K_{p, q-1} \stackrel{\eta^{(p)}}{\rightarrow} K_{p-1, q} .
$$

The following theorems constitute the theory for compact pairs, and are proved later in the section.

Theorem I. For each $(X, \alpha)$ and each $q$ the sequence

$$
\cdots \rightarrow K_{p, q} \stackrel{l}{\rightarrow} H_{p, q} \stackrel{m}{\rightarrow} K_{p, q-1} \stackrel{\eta^{(p)}}{\longrightarrow} K_{p-1, q} \stackrel{l}{\rightarrow} \cdots \rightarrow K_{0, q-1} \stackrel{\eta^{(0)}}{\longrightarrow} K_{-1, q} \rightarrow 0
$$

is exact.

Theorem II. For each $(X, \alpha)$ we have $K_{p,-1}=H_{p}(X)$ and $K_{-1, q}=H_{p}(\alpha)$.

Theorem III. The homomorphism $\eta=\eta^{(\theta)} \cdots \eta^{(p)}$ of $H_{p}(X)$ into $H_{p}(\alpha)$ given by

$$
H_{p}(X)=K_{p,-1} \stackrel{\eta^{(p)}}{\longrightarrow} K_{p-1,0} \rightarrow \cdots \rightarrow K_{0, p-1} \stackrel{\eta^{(0)}}{\longrightarrow} K_{-1, p}=H_{p}(\alpha)
$$

is such that $\eta=(-1)^{p(p+1) / 2} \pi_{\alpha}$, where $\pi_{\alpha}$ is the projection homomorphism of $H_{p}(X)$ into $H_{p}(\alpha)$.

TheOREM IV. If

$$
(X, \alpha) \stackrel{F}{\rightarrow}(Y, \beta) \stackrel{F^{\prime}}{\rightarrow}(Z, \gamma)
$$

then $\left(F^{\prime} F\right)_{h}=F_{h}^{\prime} F_{h}$ and $\left(F^{\prime} F\right)_{k}=F_{k}^{\prime} F_{k}$.

Theorem V. If $F=(f, g)$ maps $(X, \alpha)$ into $(Y, \beta)$ then $F_{k}: K_{p,-1}(\alpha) \rightarrow K_{p,-1}(\beta)$ 
coincides with $f_{*}: H_{p}(X) \rightarrow H_{p}(Y)$; also $F_{k}: K_{-1, q}(\alpha) \rightarrow K_{-1, q}(\beta)$ coincides with $g_{*}: H_{q}(\alpha) \rightarrow H_{q}(\beta)$.

THEOREM VI. If $F:(X, \alpha) \rightarrow(Y, \beta)$, there is commutativity in each rectangle of

$$
\begin{aligned}
& \cdots \rightarrow K_{p, q}(\alpha) \stackrel{l}{\rightarrow} H_{p, q}(\alpha) \stackrel{m}{\rightarrow} K_{p, q-1}(\alpha) \stackrel{\eta^{(p)}}{\rightarrow} \ldots \\
& \begin{array}{llll}
\downarrow F_{k} & \quad \downarrow F_{h} & \downarrow & \downarrow F_{k} \\
\cdots \rightarrow K_{p, q}(\beta) & \stackrel{l}{\rightarrow} H_{p, q}(\beta) & \stackrel{m}{\rightarrow} K_{p, q-1}(\beta) \longrightarrow \cdots
\end{array}
\end{aligned}
$$

We now begin the proofs.

(6.1) Lemma. Suppose that $X$ is a compact Hausdorff space, and that $\alpha=\left(A^{1}, \cdots, A^{r}\right)$ is a closed covering of $X$. If $\beta=\left(B^{1}, \cdots, B^{s}\right)$ is a collection of closed subsets of $X$, there is a collection $\gamma=\left(C^{1}, \cdots, C^{*}\right)$ of closed subsets of $X$ with $C^{i}$ containing $B^{i}$ in its interior, $i=1, \cdots, s$, and with a set of the type $C^{i_{0}} \cap \cdots \cap C^{i_{p}}$ intersecting some $A^{i}$ if and only if $B^{i_{0}} \cap \cdots \cap B^{i_{p}}$ intersects $A^{i}$.

Proof. It is sufficient to prove that if $1 \leqq k \leqq s$ then there is a $\gamma=\left(C^{1}, \ldots\right)$ $\left.C^{s}\right)$ with $C^{i}=B^{i}$ for $i \neq k, C^{k}$ containing $B^{k}$ in its interior, and $C^{i_{0}} \cap \cdots \cap C^{i_{p}}$ $\cap A^{i} \neq \varnothing$ if and only if $B^{i_{0}} \cap \cdots \cap B^{i_{p}} \cap A^{i} \neq \varnothing$. Suppose that $C^{k}$ contains $B^{k}$ in its interior and that $C^{k}$ intersects any set of the type $B^{i_{0}} \cap \cdots \cap C^{i_{p}}$ $\cap A^{i}$ if and only if $B^{k}$ intersects $B^{i_{0}} \cap \cdots \cap B^{i_{p}} \cap A^{i}$. Let $C^{i}=B^{i}$ for $i \neq k$. Then $C^{i_{0}} \cap \cdots \cap C^{i_{p}} \cap A^{i} \neq \varnothing$ if and only if $B^{i_{0}} \cap \cdots \cap B^{i_{p}} \cap A^{i} \neq \varnothing$. The assertion follows.

(6.2) Definition. Suppose that $(X, \alpha)$ is a compact pair. Then a finite open covering $u$ of $X$ is a special covering of $(X, \alpha)$ if and only if

(i) whenever $U^{i_{0}}, \cdots, U^{i_{p}}$ are elements of $u$ with $U^{i_{0}} \cap \cdots \cap U^{i_{p}}$ intersecting each of $A^{i_{0}}, \cdots, A^{i_{q}}$ then $U^{i_{0}} \cap \cdots \cap U^{i_{p}}$ intersects $A^{i_{0}} \cap \cdots \cap A^{i_{q}}$;

(ii) whenever $U^{i_{0}}, \cdots, U^{i_{p}}$ are elements of $u$ with $U^{i_{0}} \cap \cdots \cap U^{i_{p}} \neq \varnothing$, there is an element $U^{i_{k}}$ such that if $U^{i_{h}} \cap A^{i} \neq \varnothing$ then $U^{i_{0}} \cap \cdots \cap U^{i_{p}} \cap A^{j}$ $\neq \varnothing$. The set of all such special coverings will be denoted by $U(\alpha)$.

(6.3) Theorem. Suppose that $(X, \alpha)$ is a compact pair. If $v$ is an open covering of $X$, there is a special open covering $u$ of $(X, \alpha)$ which refines $v$.

Proof. Let $Y^{m}$ consist of all $x \in X$ with $x \in A^{i}$ for at least $m$ values of $i$. Then $Y^{m}$ is closed, $Y^{m+1} \subset Y^{m}$ and $Y^{1}=X$. We prove the following by induction for $r \geqq m \geqq 1$.

$L_{m}$ : there exists a covering $\beta_{m}=\left(B^{1}, \cdots, B^{*}\right)$ of $Y^{m}$ by closed subsets of $Y^{m}$ such that $\beta_{m}$ refines $v$ and such that $\beta_{m}$ satisfies (i) and (ii) of the definition of a special covering with the $U^{i}$ 's replaced by $B^{i}$ 's.

$L_{r}$ may be seen to be true. Suppose $L_{m}$ is true and that $\beta_{m}=\left(B^{1}, \cdots, B^{\star}\right)$ is the desired covering of $Y^{m}$. By Lemma 6.1, there is a collection $\gamma$ $=\left(C^{1}, \cdots, C^{\boldsymbol{b}}\right)$ of closed subsets of $Y^{m-1}$ with $C^{\boldsymbol{i}}$ containing $B^{i}$ in its interior 
relative to $Y^{m-1}$ and with $C^{i_{0}} \cap \cdots \cap C^{i_{p}}$ intersecting $A^{j}$ if and only if $B^{i_{0}} \cap \cdots \cap B^{i_{p}}$ intersects $A^{i}$. It may be seen that $\gamma$ satisfies (i) and (ii) of Definition 6.2 , since $\beta_{m}$ does. We may also suppose $\gamma$ sufficiently small so that $\gamma$ refines $v$.

Now suppose $x \in Y^{m-1}-Y^{m}$. Then $x$ is contained in exactly $m-1 A^{i}$ 's. There is also a neighborhood of $x$ relative to $Y^{m-1}$ every point of which is contained in exactly those $A^{i}$ 's which contain $x$. Hence we may expand $\gamma$ to a covering

$$
\beta_{m-1}=\left(C^{1}, \cdots, C^{s}, C^{s+1}, \cdots, C^{s+t}\right)
$$

of $Y^{m-1}$ by closed sets, where all points of $C^{*+i}$ are contained in exactly the same $A^{i^{\prime}}$ s, and where $\beta_{m-1}$ refines $v$. Suppose $C^{i_{0}} \cap \cdots \cap C^{i_{p}}$ intersects each of $A^{i_{0}}, \cdots, A^{i_{q}}$. If $i_{0}, \cdots, i_{p} \leqq s$, then $C^{i_{0}} \cap \cdots \cap C^{i_{p}}$ intersects $A^{i_{0}} \cap \cdots$ $\cap A^{i_{a}}$ since $\gamma$ satisfied (i). If some $i_{k}>s$ then $A^{i_{0}}, \cdots, A^{j_{q}}$ must each be one of the $A^{i}$ 's which contains $C^{i}{ }_{k}$. Then

$$
A^{i_{0}} \cap \cdots \cap A^{i_{q}} \supset C^{i_{k}} \supset C^{i_{0}} \cdots C^{i_{p}} .
$$

Hence $\beta_{m-1}$ satisfies (i).

Suppose now that $C^{i_{0}} \cap \cdots \cap C^{i_{p}} \neq \varnothing$. If $i_{0}, \cdots, i_{p} \leqq s$, there is a $C^{i_{k}}$ such that if $C^{i_{k}} \cap A^{j \neq} \neq \varnothing$ then $C^{i_{0}} \cap \cdots \cap C^{i_{p}} \cap A^{j} \neq \varnothing$, since $\gamma$ satisfies (ii). Suppose $i_{k}>s$. If $C^{i_{k}} \cap A^{i} \neq \varnothing$ then $A^{j} \supset C^{i_{k}}$. Then $C^{i_{0}} \cap \cdots \cap C^{i_{p}} \cap A^{i}$ $=C^{i_{0}} \cap \cdots \cap C^{i_{p}} \neq \varnothing$. Hence $\beta_{m-1}$ satisfies (ii). The induction is complete.

Now $L_{1}$ is true, with $\beta_{1}=\left(B^{1}, \cdots, B^{s}\right)$. Let $u=\left(U^{1}, \cdots, U^{s}\right)$ be such that $u$ refines $v, U^{i} \supset B^{i}$, and such that $U^{i_{0}} \cap \cdots \cap U^{i_{p}}$ intersects $A^{i}$ if and only if $B^{i_{0}} \cap \cdots \cap B^{i_{p}}$ intersects $A^{i}$. Then $u$ is a special covering, and the theorem follows.

Suppose that $(X, \alpha)$ is a compact pair. For each special covering $u \in U(\alpha)$ there is the nerve $X_{u}$. There is also a covering $\alpha_{u}=\left(A_{u}^{1}, \cdots, A_{u}^{r}\right)$ of $X_{u}$, where $A_{u}^{u}$ consists of all simplexes $\tau^{p}=\left(\tilde{U}^{0} \cdots \widetilde{U}^{p}\right)$ of $X_{u}$ with $U^{0} \cap \cdots \cap U^{p}$ $\cap A^{i} \neq \varnothing$. Furthermore, from (i) of Definition $6.2, A_{u}^{i_{0}} \cap \cdots \cap A_{u}^{i_{q}}$ consists of those simplexes $\tau^{p}$ of $X_{u}$ with $U^{0} \cap \cdots \cap U^{p}$ intersecting $A^{i_{0}} \cap \cdots \cap A^{i_{q}}$. In particular, the nerve $\left(X_{u}\right)_{\alpha_{u}}$ of $\alpha_{u}$ coincides with the nerve $X_{\alpha}$. If $T_{q}$ $=\left(\tilde{A}^{i_{0}} \cdots \tilde{A}^{i_{q}}\right)$ is a simplex of $X_{\alpha}$ then $T_{q}^{u}=\left(\tilde{A}_{u}^{i_{0}} \cdots \tilde{A}_{u}^{i_{q}}\right)$ is a simplex of $\left(X_{u}\right)_{\alpha_{u}}$ and $T_{q}=T_{q}^{u}$. Moreover, $\cap T_{q}^{u}=A_{u}^{i_{0}} \cap \cdots \cap A_{u}^{i_{q}}$ consists of all those simplices $\tau^{p}$ of $X_{u}$ with $U^{0} \cap \cdots \cap U^{p}$ intersecting $\cap T_{q}$.

If $u, v \in U(\alpha)$ and $v$ refines $u$, let $\pi_{v u}$ denote a projection of $X$, into $X_{u}$. It may be checked that $\pi_{v u}$ maps $\cap T_{q}^{0}$ into $\cap T_{q}^{u}$; this map is denoted by $\pi_{v u} \mid \cap T_{q}^{v}$. It follows that $\left(\pi_{v u}, i d\right), i d$ the identity function, maps the simplicial pair $\left(X_{v}, \alpha_{v}\right)$ into $\left(X_{u}, \alpha_{u}\right)$. Define $\tilde{F}^{v u}=\left(\pi_{v u}, i d\right)$.

(6.4) For a simplex $T_{q}$ of $X_{\alpha}$, the limit group of the inverse system $\left[H_{q}\left(\cap T_{q}^{u}\right)\right.$, $\left.\left(\pi_{v u} \mid \cap T_{q}^{*}\right)_{*}\right]$, indexed by $U(\alpha)$, is $H_{p}\left(\cap T_{q}\right)$.

Proof. We have that $\cap T_{a}^{u}$ consists of all simplices of $X_{u}$ whose intersection intersects $\cap T_{q}$. Also, $\pi_{v u} \mid \cap T_{q}^{v}$ is a projection of $\cap T_{q}^{v}$ into $\cap T_{q}^{u}$. Since $U(\alpha)$ is 
cofinal in the set of all open coverings of $X$, the limit group may be identified with $H_{p}\left(\cap T_{q}\right)$.

(6.5) If $v, u \in U(\alpha)$ and $v$ refines $u$, then $\left(\widetilde{F}^{v u}\right)_{h}=\widetilde{F}_{h}^{o u}$ and $\left(\widetilde{F}^{v u}\right)_{k}=\widetilde{F}_{\boldsymbol{k}}^{o u}$ are independent of the projection $\pi_{v u}$. If also $w$ refines $v$ then $\tilde{F}_{h}^{o u} \tilde{F}_{h}^{w o v}=\tilde{F}_{h}^{a v u}$ and $\tilde{F}_{k}^{o u} \tilde{F}_{k}^{\text {voo }}=\tilde{F}_{k}^{\text {cou }}$.

Proof. If $\pi_{v u}$ and $\pi_{v u}^{\prime}$ are projections of $X_{v}$ into $X_{u}$, then the maps $\left(\pi_{v u}, i d\right)$ and $\left(\pi_{v u}^{\prime}, i d\right)$ satisfy the hypothesis of (5.8). Hence $F_{h}^{o u}$ and $F_{k}^{o u}$ are independent of the projection.

(6.6) The limit group of the inverse system $\left[H_{p, q}\left(\alpha_{u}\right), \widetilde{F}_{h}^{o u}\right]$, indexed by $U(\alpha)$, is isomorphic with $H_{p, q}(\alpha)$. Henceforth we identify the two.

Proof. Consider an element $A_{p} \cdot T_{q}$ of $H_{p, q}(\alpha)$, where $A_{p} \in H_{p}\left(\cap T_{q}\right)$. According to (6.4), $A_{p}=\left(A_{p}(u): u \in U(\alpha)\right)$, where $A_{p}(u)$ is the coordinate of $A_{p}$ in $H_{p}\left(\cap T_{q}^{u}\right)$, and where, if $v$ refines $u$, then $\left(\pi_{v u} \mid \cap T_{q}^{v}\right)_{*} A_{p}(v)=A_{p}(u)$. For each $v, A_{p}(v) \cdot T_{q}^{v}$ is an element of $H_{p, q}\left(\alpha_{v}\right)$ and if $v$ refines $u$ then $\widetilde{F}_{h}^{o u}$ maps $A_{p}(v) \cdot T_{q}^{v}$ into $A_{p}(u) \cdot T_{q}^{u}$. The correspondence

$$
A_{p} \cdot T_{q} \rightarrow\left(A_{p}(u) \cdot T_{q}^{u}: u \in U(\alpha)\right)
$$

together with linearity, yields an isomorphism of $H_{p, q}(\alpha)$ onto the limit group.

(6.7) Definition. For a pair $(X, \alpha), K_{p, q}(\alpha)$ is defined to be the limit group of the inverse system $\left[K_{p, q}\left(\alpha_{u}\right), \tilde{F}_{k}^{\text {ou }}\right]$, indexed by $U(\alpha)$. The maps

$$
K_{p, q}(\alpha) \stackrel{l}{\rightarrow} H_{p, q}(\alpha) \stackrel{m}{\rightarrow} K_{p, q-1} \stackrel{\eta^{(p)}}{\rightarrow} K_{p-1, q}(\alpha)
$$

are defined as limits of

$$
K_{p, q}\left(\alpha_{u}\right) \stackrel{l}{\rightarrow} H_{p, q}\left(\alpha_{u}\right) \stackrel{m}{\rightarrow} K_{p, q-1} \stackrel{\eta^{(p)}}{\longrightarrow} K_{p-1, q}\left(\alpha_{u}\right) .
$$

Theorem I then follows from (5.5), together with the fact that the groups of $\alpha_{u}$ are either compact or finite-dimensional vector spaces.

THEOREM II. We have $K_{p,-1}(\alpha)=H_{p}(X), K_{-1, q}(\alpha)=H_{q}(\alpha)$.

Proof. Consider the inverse system $\left[K_{p,-1}\left(\alpha_{u}\right), \widetilde{F}_{k}^{p u}\right]$. According to (5.3), $K_{p,-1}\left(\alpha_{u}\right)=H_{p}(u)$. According to $(5.7), F_{k}: K_{p,-1}\left(\alpha_{v}\right) \rightarrow K_{p,-1}\left(\alpha_{u}\right)$ coincides with $\pi_{v u}: H_{p}(v) \rightarrow H_{p}(u)$. Hence the inverse system coincides with $\left[H_{p}(u)\right.$, $\left.\pi_{v u}\right]$ and $K_{p,-1}=H_{p}(X)$. Consider now the inverse system $\left[K_{-1, q}\left(\alpha_{u}\right), \tilde{F}_{k}^{o u}\right]$. Now $K_{-1, q}\left(\alpha_{u}\right)=H_{q}\left(\left(X_{u}\right) \alpha_{u}\right)=H_{q}(u)$ and $\tilde{F}_{k}^{m u}$ is the identity by (5.7). Hence $K_{-1, q}$ may be identified with $H_{q}(\alpha)$.

THEOREM III. The homomorphism $\eta: H_{p}(X) \rightarrow H_{p}(\alpha)$ is such that $\eta$ $=(-1)^{p(p+1) / 2} \pi_{\alpha}$.

Proof. Let $W$ be an open covering $\left(W^{0}, \cdots, W^{r}\right)$ of $X$ where $W^{i} \supset A^{\boldsymbol{i}}$ and where $W^{i_{0}} \cap \cdots \cap W^{i_{q}} \neq \varnothing$ if and only if $A^{i_{0}} \cap \cdots \cap A^{i_{q}} \neq \varnothing$. Then $X_{w}=X_{\alpha}$ and $\pi_{w}=\pi_{u}$. Consider any special covering $u$ of $X$ such that if $U$ is 
an element of $u$ and $U \cap A^{i} \neq \varnothing$ then $U \subset W^{i}$. Since $u$ is a special covering, if $U^{0} \cap \cdots \cap U^{p} \neq \varnothing$, where the $U^{i}$ s are elements of $u$, there is an element $U^{i}$ such that if $U^{i} \cap A^{j} \neq \varnothing$ then $U^{0} \cap \cdots \cap U^{p} \cap A_{j} \neq \varnothing$. It follows that $\left(X_{u}\right.$, $\alpha_{u}$ ) satisfies the hypothesis of (5.6). Let $f$ be a function assigning to each vertex $U$ of $X_{u}$ an element $A_{u}^{i}$ of $\alpha_{u}$ with $U \cap A^{i} \neq \varnothing$ (that is, $U$ is a vertex of $\left.A_{u}^{i}\right)$. According to (5.6), $f: X_{u} \rightarrow\left(X_{u}\right) \alpha_{u}=X_{\alpha}$ is simplicial and $f_{*}: H_{p}\left(X_{u}\right)$ $\rightarrow H_{p}\left(\left(X_{u}\right) \alpha_{u}\right)$ is such that $f_{*}=(-1)^{p(p+1) / 2} \eta_{u}$ where $\eta_{u}$ is the composition

$$
H_{p}\left(X_{u}\right)=K_{p,-1}\left(\alpha_{u}\right) \stackrel{\eta^{(p)}}{\longrightarrow} \cdots \stackrel{\eta^{(0)}}{\longrightarrow} K_{-1, p}\left(\alpha_{u}\right)=H_{p}\left(\left(X_{u}\right) \alpha_{u}\right) .
$$

In the diagram

$$
X_{u} \stackrel{f}{\rightarrow}\left(X_{u}\right) \alpha_{u}=X_{\alpha}=X_{w}
$$

note that $u$ and $w$ are chosen so that $f: X_{u} \rightarrow X_{w}$ is a projection $\pi_{u w}$ of $X_{u}$ into $X_{w}$. So $\pi_{u w *}=f_{*}$ and

$$
\pi_{u w *}=(-1)^{p(p+1) / 2} \eta_{u}, \quad \pi_{w}=(-1)^{p(p+1) / 2} \eta .
$$

Since $X_{\alpha}=X_{w}$ and $\pi_{\alpha}=\pi_{w}$, the theorem follows.

(6.8) Definition. Suppose $F=(f, g)$ maps the compact pair $(X, \alpha)$ into the compact pair $(Y, \beta)$ where $\beta=\left(B^{1}, \cdots, B^{s}\right)$. Denote by $P(F)$ the set of all pairs $(u, v)$, where $u \in U(\alpha)$ and $v \in U(\beta)$ and where if $U$ is an element of $u$ then there is an element $V$ of $v$ with $f(U) \subset V$. Let $f_{u v}: X_{u} \rightarrow X_{v}$ assign to each vertex $U$ of $X_{u}$ a vertex $V$ of $Y_{v}$ with $f(U) \subset V$. Then $f_{u v}$ is simplicial.

There are the coverings $\alpha_{u}=\left(A_{u}^{1}, \cdots, A_{u}^{r}\right)$ of $X$ and $\beta_{v}=\left(B_{v}^{1}, \cdots, B_{v}^{s}\right)$ of $Y_{v}$. Moreover $\left(X_{u}\right) \alpha_{u}=X_{\alpha}$ and $\left(Y_{v}\right) \beta_{v}=Y_{\beta}$. It may be seen that $F^{u v}=\left(f_{u v}, g\right)$ maps the pair $\left(X_{u}, \alpha_{u}\right)$ into the pair $\left(Y_{v}, \beta_{v}\right)$. Moreover, by (5.8), $F_{h}^{u v}: H_{p, q}\left(\alpha_{u}\right)$ $\rightarrow H_{p, q}\left(\beta_{v}\right)$ and $F_{k}^{u v}$ are independent of the particular $f_{u v}$ chosen. Also, if $u^{\prime} \in U(\alpha)$ and $u^{\prime}$ refines $u$ we have $\widetilde{F}^{u^{\prime} u}=\left(\pi_{u^{\prime} u}, i d\right)$ mapping $\left(X_{u^{\prime}}, \alpha_{u^{\prime}}\right)$ into $\left(X_{u}, \alpha_{u}\right)$. If $v$ refines $v^{\prime} \in U(\beta)$ there is $F^{v v^{\prime}}=\left(\pi_{v v^{\prime}}, i d\right)$ mapping $\left(Y_{v}, \beta_{v}\right)$ into $\left(Y_{v^{\prime}}, \beta_{v^{\prime}}\right)$. Then $\tilde{F}^{v v^{\prime}} F^{u v} \widetilde{F}^{u^{\prime} u}=F^{u^{\prime} v^{\prime}}$. Hence

$$
\widetilde{F}_{h}^{v v^{\prime}} F_{h}^{u v} \widetilde{F}_{h}^{u^{\prime} v}=F_{h}^{u^{\prime} v^{\prime}}, \quad \widetilde{F}_{k}^{v v^{\prime}} F_{k}^{u v} \widetilde{F}_{k}^{u^{\prime} u}=F_{k}^{u^{\prime} v^{\prime}} .
$$

Consider the inverse systems $\left[K_{p, q}\left(\alpha_{u}\right), \tilde{F}_{k}^{\alpha^{\prime} u}\right]$, indexed by $U(\alpha)$, and $\left[K_{p, q}\left(\beta_{v}\right), \tilde{F}_{\boldsymbol{k}}^{c \sigma^{\prime}}\right]$, indexed by $U(\beta)$. For $(u, v) \in P(F)$, there is the map $F_{k}^{\text {vu }}: K_{p, q}\left(\alpha_{u}\right) \rightarrow K_{p, q}\left(\beta_{v}\right)$ such that $\left(^{*}\right)$ holds. There is induced, in the limit, a map $F_{k}: K_{p, q}(\alpha) \rightarrow K_{p, q}(\beta)$.

(6.9) The limit of the map $F_{h}^{u v}: H_{p, q}\left(\alpha_{u}\right) \rightarrow H_{p, q}\left(\beta_{v}\right),(u, v) \in P(F)$, is the map $F_{h}$ defined earlier.

Proof. It is sufficient to check the equality of the two maps on elements $A_{p} \cdot T_{q}, T_{q}$ an oriented $q$-simplex of $\alpha$ and $A_{p} \in H_{p}\left(\cap T_{q}\right)$. Now $F_{h}$ maps $A_{p} \cdot T_{q}$ into $\left[\left(f \mid \cap T_{q}\right)_{*} A_{p}\right] \cdot g T_{q}$. Also, according to $(6.6), A_{p} \cdot T_{q}=\left(A_{p}(u) \cdot T_{q}^{u}\right.$ : $u \in U(\alpha))$. Now $F_{h}^{\text {ru }}$ maps $A_{p}(u) \cdot T_{q}^{u}$ into $\left[\left(f_{u v} \mid \cap T_{q}^{u}\right)_{*} A_{p}(u)\right] \cdot g T_{q}^{u}$. This is the coordinate in $H_{p . q}\left(\beta_{v}\right)$ of $\left[\left(f \mid \cap T_{q}\right)_{*} A_{p}\right] \cdot g T_{q}$. 
We leave the proofs of Theorems IV, V, and VI to the reader.

\section{Proof of the basic theorem.}

Theorem. Suppose that $A_{-1} \supset A_{0} \supset \cdots \supset A_{n}$ is a sequence of closed subsets of a compact space $X$, and that $\alpha_{-1} \ll^{n} \ldots \ll^{n} \alpha_{n}$, where $\alpha_{i}$ is a closed covering of $A^{i}$. Then

(i) the kernel of $\pi_{\alpha_{n}}: H_{j}\left(A_{n}\right) \rightarrow H_{j}\left(\alpha_{n}\right)$ is contained in the kernel of the injection $I: H_{j}\left(A_{n}\right) \rightarrow H_{j}\left(A_{-1}\right)$ for $j \leqq n$;

(ii) the image of $\pi_{\alpha_{n}, \alpha_{0}}: H_{j}\left(\alpha_{n}\right) \rightarrow H_{j}\left(\alpha_{0}\right)$ is contained in the image of $\pi_{\alpha_{0}}$ : $H_{j}\left(A_{0}\right) \rightarrow H_{j}\left(\alpha_{0}\right)$ for $j \leqq n+1$.

Proof. For each $i$ there is a projection $\pi=\pi_{\alpha_{i}, \alpha_{i-1}}$ of $\alpha_{i}$ into $\alpha_{i-1}$ such that $H_{j}\left(A^{i_{0}} \cap \cdots \cap A^{i_{q}} ; \pi A^{i_{0}} \cap \cdots \cap \pi A^{i_{q}}\right)=0$ for all $j \leqq n$. Let $I_{i}^{\prime}: A_{i} \rightarrow A_{i-1}$ denote the inclusion map. Then $F^{i}=\left(I_{i}^{\prime}, \pi\right)$ maps $\left(A_{i}, \alpha_{i}\right)$ into $\left(A_{i-1}, \alpha_{i-1}\right)$ and $F_{h}^{i}: K_{p, q}\left(\alpha_{i}\right) \rightarrow K_{p, q}\left(\alpha_{i-1}\right)$ is trivial for $0 \leqq p \leqq n$.

Consider the diagram

$$
\begin{aligned}
& \cdots \rightarrow H_{p, q}\left(\alpha_{i}\right) \stackrel{m}{\longrightarrow} K_{p, q-1}\left(\alpha_{i}\right) \stackrel{\eta^{(p)}}{\longrightarrow} K_{p-1, q}\left(\alpha_{i}\right) \stackrel{l}{\longrightarrow} H_{p-1, q}\left(\alpha_{i}\right) \longrightarrow \cdots \\
& \downarrow F_{h}^{i} \quad \downarrow F_{k}^{i} \quad \downarrow F_{k}^{i} \quad, \quad \downarrow F_{h}^{i} \\
& \cdots \rightarrow H_{p, q}\left(\alpha_{i-1}\right) \stackrel{m}{\rightarrow} K_{p, q-1}\left(\alpha_{i-1}\right) \stackrel{\eta^{(p)}}{\rightarrow} K_{p-1, q}\left(\alpha_{i-1}\right) \stackrel{l}{\rightarrow} H_{p-1, q}\left(\alpha_{i-1}\right) \rightarrow \cdots
\end{aligned}
$$

Now (1) if $x \in K_{p, q-1}\left(\alpha_{i}\right), p \leqq n$, and $\eta^{(p)}(x)=0$, then $F_{k}^{i}(x)=0$. For if $\eta^{(p)}(x)=0$ then $x=m(y)$ and $F_{k}^{i}(x)=F_{k}^{i} m(y)=m F_{h}^{i}(y)=0$. Moreover, (2) if $x \in K_{p-1, q}\left(\alpha_{i}\right), p \leqq n+1$, then $F_{k}^{i}(x) \in K_{p-1, q}\left(\alpha_{i-1}\right)$ is of the form $\eta^{(p)}(y)$ for some $y \in K_{p, q-1}\left(\alpha_{i-1}\right)$.

To prove (i), suppose $\pi_{\alpha_{n}}(x)=0, x \in H_{j}\left(A_{n}\right)=K_{j,-1}\left(\alpha_{n}\right)$. Then, by Theorem III, $\eta(x)=\eta^{(0)} \cdots \eta^{(j)}(x)=0$ in $K_{-1, j}\left(\alpha_{n}\right)=H_{j}\left(\alpha_{n}\right)$. By (1), $F_{k}^{n} \eta^{(1)} \cdots$ $\eta^{(j)}(x)=\eta^{(1)} \cdots \eta^{(j)} F_{k}^{n}(x)=0$ in $K_{0, j-1}\left(\alpha_{n-1}\right)$. By (1) again, $F_{k}^{n-1} \eta^{(2)} \cdots$ $\eta^{(j)} F_{k}^{n}(x)=\eta^{(2)} \cdots \eta^{(j)} F_{k}^{n-1} F_{k}^{n}(x)=0$ in $K_{1, j-2}\left(\alpha_{n-2}\right)$. Continuing, $F_{k}^{n-j} \cdots$ $F_{k}^{n-1} F_{k}^{n}(x)=0$ in $K_{j,-1}\left(\alpha_{n-j-1}\right)$. But $F_{k}^{n-j} \cdots F_{k}^{n}(x)$ is, by Theorem V, the injection $I$ of $H_{j}\left(A_{n}\right)$ into $H_{j}\left(A_{n-j-1}\right)$. Hence (i) follows.

To prove (2), suppose $x \in H_{j}\left(\alpha_{n}\right)=K_{-1, j}\left(\alpha_{n}\right)$. The map

$$
K_{0, j-1} \stackrel{\eta^{(0)}}{\longrightarrow} K_{-1, j}
$$

is onto, by Theorem I, so $x=\eta^{(0)}\left(x_{0}\right), x_{0} \in K_{0, j-1}\left(\alpha_{n}\right)$. By (2), $F_{k}^{n}\left(x_{0}\right)$ $\in K_{0, j-1}\left(\alpha_{n-1}\right)$ is of the form $\eta^{(1)}\left(x_{1}\right)$ for some $x_{1} \in K_{1, j-2}\left(\alpha_{n-1}\right)$. Continuing by use of (2), $F_{k}^{n-j+2}\left(x_{j-1}\right) \in K_{j-1,0}\left(\alpha_{n-j+1}\right)$ is of the form $\eta^{(j)}\left(x_{j}\right)$ for some $x \in K_{j,-1}\left(\alpha_{n-j+1}\right)$. Then

$$
\begin{aligned}
\eta^{(0)} \cdots \eta^{(j)}\left(x_{j}\right) & =\eta^{(0)} \cdots \eta^{(j-1)} F_{k}^{n-j+2}\left(x_{j-1}\right) \\
& =F_{k}^{n-j+2} \eta^{(0)} \cdots \eta^{(j-2)} F_{k}^{n-j+3}\left(x_{j-2}\right) \\
& =F_{k}^{n-j+2} \cdots F_{k}^{n}(x) .
\end{aligned}
$$


By Theorems IV and V, $F_{k}^{n-\jmath+2} \cdots F_{k}^{n}$ is the projection $\pi$ of $H_{j}\left(\alpha_{n}\right)$ into $H_{j}\left(\alpha_{n-j+1}\right)$. Hence image $\pi_{\alpha_{n}, \alpha_{n-j+1}}$ Cimage $\pi_{\alpha_{n-j+1}}$. For $j \leqq n+1$, it follows readily that image $\pi_{\alpha_{n}, \alpha_{0}} \subset$ image $\pi_{\alpha_{0}}$.

\section{BIBLIOGRAPHY}

1. E. G. Begle, The Vietoris mapping theorem for bicompact spaces, Ann. of Math. vol. 51 (1950) pp. 534-543.

2. S. Eilenberg and N. E. Steenrod, Foundations of algebraic topology, Princeton, 1952.

3. J. L. Kelley and E. Pitcher, Exact homomorphism sequences in homology theory, Ann. of Math. vol. 48 (1947) pp. 682-709.

4. P. A. White, Regular convergence, Bull. Amer. Math. Soc. vol. 60 (1954) pp. 431-443. 432.

5. - Regular convergence in terms of Cech cycles, Ann. of Math. vol. 55 (1952) pp. 420-

6. R. L. Wilder, Topology of manifolds, New York, 1949.

UNIVERSITY OF VIRGINIA,

Charlottesville, Va. 